(c) 2021 Universidad Nacional Autónoma de México, Facultad de Estudios Superiores Zaragoza.

Este es un artículo Open Access bajo la licencia CC BY-NC-ND (http://creativecommons.org/licenses/by-nc-nd/4.0/).

TIP Revista Especializada en Ciencias Químico-Biológicas, 24: 1-15, 2021.

https://doi.org/10.22201/fesz.23958723e.2021.365

\title{
Elaboración de un envase primario para alimentos a partir de residuos de maíz y piñón mexicano
}

\author{
Alejandra Linares-Castañeda, Luis Jorge Corzo-Ríos, \\ Esther Bautista-Ramírez y Yolanda de las Mercedes Gómez y Gómez* \\ Unidad Profesional Interdisciplinaria de Biotecnología, Instituto Politécnico Nacional, \\ Av. Acueducto s/n, Col. Barrio La Laguna Ticomán 07340, Ciudad de México, México. \\ E-mail: *ygomez@ipn.mx
}

\begin{abstract}
RESUMEN
El creciente interés por conservar el medio ambiente ha motivado el desarrollo de envases biodegradables para alimentos a partir de residuos agroindustriales. Los cultivos de maíz y piñón mexicano generan cantidades considerables de residuos lignocelulósicos que pueden emplearse como materia prima para elaborar envases de papel. El objetivo de este trabajo fue elaborar y caracterizar un envase de papel a partir de la celulosa de residuos fibrosos de piñón mexicano (CCJ) y de tallos/hojas de maíz (CTM) para determinar su funcionalidad en la prolongación de la vida útil de chiles serranos almacenados a temperatura ambiente. Se elaboró papel a partir de diferentes concentraciones (100-0 a 80-20\%) de CTM y CCJ por método alcalino con $\mathrm{NaOH}$. Se seleccionó la formulación de papel P5 correspondiente a una relación 95-5\% de CTM-CCJ respectivamente con la finalidad de emplearlo para preservar la calidad del chile serrano (gramaje $97.0 \mathrm{~g} / \mathrm{m}^{2}$, dureza $3112 \mathrm{MPa}$ y espesor $0.25 \mathrm{~mm}$ ). El uso de P5 como envase para chile serrano favorece los valores de firmeza, humedad, color y vitamina $\mathrm{C}$, con respecto a los chiles testigo durante los días de almacenamiento a temperatura ambiente. Las propiedades de textura y color son sumamente importantes en la calidad cualitativa del chile serrano, al considerar estos criterios en la adquisición del producto para su consumo en fresco.
\end{abstract}

Palabras clave: envase de papel, maíz, piñón mexicano, chile serrano, almacenamiento postcosecha.

\section{Elaboration of a primary packaging for food from \\ residues of corn and mexican pinion}

\begin{abstract}
The growing interest in conserving the environment is motivating the development of biodegradable packaging for food from agro-industrial waste. Corn crops and Mexican pinion generate considerable amounts of lignocellulose waste that can be used as feedstock to make paper packaging. The objective of this work was to elaborate and characterize a paper packaging from cellulose of fibrous residues of Mexican pinion (CCJ) and corn stem/leaves (CTM), and to determine its functionality in prolonging the useful life of serrano peppers stored at room temperature. Paper was made from different concentrations (100-0 to 80-20\%) of CTM and CCJ by alkaline method with $\mathrm{NaOH}$. Paper formulation $\mathrm{P} 5$ was selected corresponding to a relation $95-5 \%$ of CTM-CCJ respectively with the purpose to use it to preserve quality in Serrano pepper (grammage $97.0 \mathrm{~g} / \mathrm{m}^{2}$, hardness $3112 \mathrm{MPa}$, thickness $0.25 \mathrm{~mm}$ ). The use of P5 as packaging for Serrano pepper favors the values of firmness, moisture, color and vitamin $\mathrm{C}$ with respect to the control pepper during the days of storage at room temperature. The properties of texture and color are extremely important in the qualitative quality of Serrano pepper, since being a variety of fresh consumption, are criteria for consumption or acquisition of this product. Keywords: paper packaging, corn, mexican pinion, serrano pepper, postharvest storage.
\end{abstract}

Artículo recibido el 15 de mayo del 2021.

Artículo aceptado el 04 de octubre del 2021. 


\section{INTRODUCCIÓN}

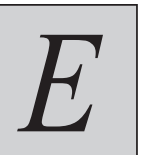

1 continuo desarrollo de la industria de embalaje y logística, aumenta la demanda en el mercado del papel año tras año (Xia et al., 2019). La celulosa es el principal constituyente de la madera, por lo que es un recurso natural renovable de fácil acceso que se ha utilizado durante los últimos 150 años en la elaboración de materiales de uso cotidiano (Sethi, Oksman, Illikainen \& Sirviö, 2018). Existen otras fuentes importantes de celulosa como en las fibras vegetales, presente en la hoja, fruto y en la estructura rígida de las plantas (algodón, cáñamo, lino, etc.), en los animales marinos (tunicados) o las algas, los hongos, los invertebrados y en las bacterias (Lavoine, Desloges, Dufresne \& Bras, 2012).

Los residuos de cultivos agrícolas son una fuente abundante y barata de celulosa que se han adecuado para la elaboración de textiles, pulpa y papel. El uso de fibras no maderables para la producción del papel es importante para reducir su consumo con la opción de llevar a cabo nuevas aplicaciones a partir de los residuos mencionados utilizándolos como materia prima. La paja de canola (Yousefi et al., 2013), los tallos de maíz (Fan \& Zhang, 2018), la cáscara de arroz y el bagazo de caña (Bras et al., 2010), entre otros, son subproductos agrícolas renovables anualmente y están disponibles en abundancia en todo el mundo.

El interés en estos subproductos ricos en fibra a base de celulosa está aumentando debido a su viabilidad como fuente de fibra natural en países con escases de recursos madereros, por sus beneficios medioambientales debido a su naturaleza renovable y su bajo consumo de energía para su producción (Abrantes, Amaral, Costa \& Duarte, 2007; Adel, El-Gendy, Diab, AbouZeid \& El-Zawawy, 2016).

El piñón mexicano (Jatropha curcas) es una planta tropical nativa de México y América central, es perene y atrajo la atención mundial como una fuente alternativa de energía sostenible debido principalmente, a su alto contenido de aceite en su semilla no comestible (Mazumdar, Singh, Babu, Siva \& Harikrishna, 2018), que ha cobrado importancia en la producción del biodiesel (Kumar \& Sharma, 2008; Pandey et al., 2012).

La semilla contiene aproximadamente entre el $58-60 \%$ de granos y entre el $40-42 \%$ de cáscara; se estima que se producen 2.4 Ton de cáscara para obtener $350 \mathrm{~L}$ de aceite. La cáscara es un residuo compuesto por lignina, celulosa y hemicelulosa; sin embargo, no tiene mucho valor como forraje, fertilizante o como material combustible (leña o carbón), por lo que es importante generar valor agregado para que el uso general del piñón mexicano tenga mayor viabilidad en el mercado (Lim, Shamsudin, Baharudin, \& Yunus, 2015; Pandey et al., 2012).

Por otra parte, el maíz (Zea mays) es miembro de la familia de las gramíneas (Eckhoff, Paulsen \& Yang, 2003), es una planta anual cultivada en todo el mundo (Pechanova \& Pechan, 2017) que genera grandes cantidades de desechos en su producción. Estos subproductos se cortan y se pueden usar para forraje, sin embargo, se dejan en el campo o son quemados por los agricultores, lo que ocasiona daños al medio ambiente. Por otra parte, la investigación muestra que estos residuos (tallos y hojas) del maíz pueden usarse en otras aplicaciones debido a su composición lignocelulósica, para elaborar películas o papel (Egüés, Sánchez, Mondragón \& Labidi, 2012; Han et al., 2019; Jarabo, Monte, Fuente, Santos \& Negro, 2013; Liu, Wang, Lin, Zhang \& Xiao, 2018; Liu et al., 2019; Luo et al., 2017; Xia et al., 2019).

Adicionalmente, las pérdidas postcosecha de frutos y verduras se refieren a las pérdidas que ocurren en el manejo, almacenamiento, transporte y procesamiento, cuyo resultado es una reducción en la cantidad, calidad y valor en el mercado (Emana et al., 2017). Por otra parte, el desperdicio de alimentos se produce al final de la cadena de suministro, donde son incluidos el comercio minorista y el consumo. Las pérdidas postcosecha se pueden dividir en cualitativas (calidad percibida en términos de color, tamaño, forma, sabor, etc.) y cuantitativas (pérdidas destructivas por deterioro fisiológico, mecánico o patológico) (Porat, Lichter, Terry, Harker \& Buzby, 2018). En particular, la coloración, la frescura y la firmeza son los atributos de gran importancia para los consumidores (Rao, Gol \& Shah, 2011).

El chile serrano (Capsicum annuum acuminatum) es uno de los cultivares dentro de este género más comunes e importantes de México (Anguiano Barrales, J., 2010; Florentino-Ramos et al., 2019; Villa-Ruano et al., 2019). Además, es considerado un cultivo ampliamente utilizado, sin embargo, es una verdura muy perecedera con una vida útil corta (Chitravathi, Chauhan \& Raju, 2014).

Los chiles se deterioran rápidamente debido a una mala manipulación y almacenamiento después de la cosecha, los problemas más comunes son la degradación de su calidad, daños ocasionados por el frío, marchitamiento asociado a una rápida pérdida de peso y cambios en su color en condiciones ambientales (Chitravathi, Chauhan, \& Raju, 2015). Se han empleado diversas estrategias de envasado para prolongar la vida de anaquel de algunos tipos de chiles del género Capsicum annuиm. Entre estas estrategias se han implementado recubrimientos de ácido giberélico, goma arábiga, quitosano o envasados en atmósferas modificadas (Chitravathi, Kallepalli, Chauhan \& Kizhakkedath, 2020; Hedayati \& Niakousari, 2015; Hernández-López, VenturaAguilar, Correa-Pacheco, Bautista-Baños \& Barrera-Necha, 2020; Panigrahi, Gheewala, Patel, Patel \& Gantait, 2017; Singh, Giri \& Kotwaliwale, 2014; Xing et al., 2011). Sin embargo, la mayoría de los agricultores, transportistas y consumidores no pueden pagar los costos de mantenimiento de las instalaciones de almacenamiento atmosférico controlado o implementar el empleo de recubrimientos o películas comestibles, por lo que es necesario desarrollar sistemas económicos y viables para 
conservar los atributos postcosecha de los productos perecederos (Adetunji, Ojediran, Adetunji \& Owa, 2019).

Por lo anterior, los residuos lignocelulósicos del piñón mexicano y del maíz pueden utilizarse y estudiarse como materia prima para la elaboración de envases de papel para alimentos como sistemas sostenibles asumiendo el desafío de lo que esto implica, y siempre respetuosos con el medio ambiente para reducir las pérdidas de alimentos. El objetivo de este trabajo fue elaborar y caracterizar un envase de papel a partir de la celulosa de residuos fibrosos del piñón mexicano (CCJ) y de tallos/hojas de maíz (CTM), con el fin de determinar su funcionalidad y prolongar la vida útil de los chiles serranos almacenados a temperatura ambiente.

\section{Materiales Y MÉTODOS}

\section{Análisis químico proximal}

La caracterización químico proximal del tallo/hoja de maíz, así como de la cáscara del piñón se realizó según los métodos de la AOAC (1997). Se usó el método 925,09 de la AOAC (Association of Official Analytical Chemists, por sus siglas en inglés) para la determinación de la humedad por pérdida de peso después de secar las muestras a $105^{\circ} \mathrm{C}$ por $4 \mathrm{~h}$. La grasa cruda (método 920,39), fue cuantificada por extracción de la muestra durante 1 hora con hexano en un sistema Soxhlet. La proteína cruda (método 955,04) se determinó por el método de microKjeldahl, usando un factor de conversión de nitrógeno a proteína de 6,25. Por otro lado, la fibra cruda (método 962,09), se calculó después de la digestión ácida y alcalina. Finalmente, las cenizas (método 923,03), se obtuvieron como el peso remanente después de calcinar la muestra en una mufla a $550{ }^{\circ} \mathrm{C}$ por 4 horas. El extracto libre de nitrógeno (ELN) se obtuvo por diferencia al $100 \%$ del resto de los constituyentes.

\section{Obtención de la celulosa de las hojas y los tallos del maíz} (CTM) y cáscara del piñón mexicano (CCJ)

Se redujo el tamaño de las hojas y los tallos previamente secos, así como de la cáscara del piñón, con el uso de una licuadora comercial (Oster, MOD. 465-15) hasta que la muestra pasó por un tamiz (malla No. 30) y se procedió de acuerdo a la metodología establecida por Fan \& Zhang (2018) con algunas modificaciones. Se mezclaron $50 \mathrm{~g}$ de muestra con $300 \mathrm{~mL}$ de $\mathrm{NaOH}$ al 3\% (10\% para CCJ) y se llevó a una temperatura de $80^{\circ} \mathrm{C}$ durante 1 hora con agitación constante. Posteriormente, se enfrió a temperatura ambiente y la pulpa se lavó con abundante agua hasta $\mathrm{pH}$ neutro.

\section{Elaboración del papel}

Se siguió la metodología reportada por Iewkittayakorn et al. (2020) con ligeras modificaciones. Se tomaron las proporciones $(\mathrm{p} / \mathrm{p})$ correspondientes de CTM y de CCJ descritas en el Cuadro I, se mezclaron y la mezcla se distribuyó uniformemente sobre una malla de nylon de $48 \mu \mathrm{m}$, dejándola secar a $50{ }^{\circ} \mathrm{C} 12 \mathrm{~h}$ en una estufa de convección forzada (BG

\section{Cuadro I. Proporción de la fibra de CCJ y CTM en la elaboración de los papeles (p/p).}

\begin{tabular}{|c|c|c|c|c|c|}
\hline \multirow{2}{*}{$\begin{array}{c}\text { Fuente de } \\
\text { Celulosa }\end{array}$} & \multicolumn{5}{|c|}{ Formulaciones de papel } \\
\cline { 2 - 6 } & P1 & P2 & P3 & P4 & P5 \\
\hline CCJ & 0 & 20 & 10 & 10 & 5 \\
\hline CTM & 100 & 80 & 90 & 90 & 95 \\
\hline
\end{tabular}

Instrumentos analíticos, México) para finalmente desprenderla cuidadosamente. Las formulaciones para el papel 3 y 4 se elaboraron con las mismas proporciones 10:90 (CCJ:CTM) la diferencia fue que tanto las formulaciones del P4 como del P5 se tamizaron en una malla No. 50 y la formulación P5 fue posteriormente prensada (colocando un peso de $885 \mathrm{~g}$ sobre un área de $295 \mathrm{~cm}^{2}$ ).

\section{Características del papel}

Se determinaron las principales características físicas de cada papel elaborado, así como al papel de uso comercial (P6). Gramaje: se siguió la metodología establecida por la NMX-N-001-SCFI (2011). Dureza: se midió la fuerza necesaria para romper los papeles, la prueba se realizó con un penetrómetro en tres puntos diferentes para cada uno. Los valores se reportaron en MPa. Espesor: el espesor se midió en al menos tres puntos diferentes para cada papel reportándose en mm (Iewkittayakorn et al., 2020). Humedad: se siguió la metodología establecida por la NMX-N-016-SCFI (2011). Absorción de agua: se siguió la metodología establecida por la NMX-N-098-SCFI (2014). Observación microscópica: se observó el alineamiento de las fibras de cada papel utilizando un microscopio óptico con un objetivo de 4/0.10.

\section{Evaluación del papel como envase para el chile serrano}

Los chiles con madurez comercial fueron adquiridos en la central de abastos de la Ciudad de México, y se desinfectaron en una solución de $200 \mathrm{ppm}$ de hipoclorito de sodio $(\mathrm{NaClO})$ durante 2 minutos.

Se envolvieron los chiles individualmente con el papel seleccionado previamente (P5) excepto los testigo. Se almacenaron a $20 \pm 1{ }^{\circ} \mathrm{C}$ y $63.4 \pm 2.51 \% \mathrm{HR}$, y se determinaron los cambios en sus principales características fisicoquímicas.

Firmeza: se perforó cada fruto. Se utilizó un probador de dureza de la fruta LUTRON (modelo FR5130) (EE.UU.). La firmeza se midió cada $24 \mathrm{~h}$ por 10 días, reportando el valor medio de las mediciones (Hernández-López et al., 2020).

Acidez titulable: se determinó de acuerdo con la metodología reportada por Miranda-Molina et al. (2019) con ligeras modificaciones, se pesaron $25 \mathrm{~g}$ del producto molido y se añadieron $250 \mathrm{~mL}$ de agua destilada. Las muestras se titularon con $\mathrm{NaOH} 0.1 \mathrm{~N}$. 
pH: se determinó de acuerdo con la metodología reportada por Valiathan \& Athmaselvi (2018) con ligeras modificaciones. Se colocaron aproximadamente $100 \mathrm{~g}$ del producto en un mortero, se adicionaron $20 \mathrm{~mL}$ de agua destilada y se filtró la muestra. Posteriormente se sumergieron los electrodos en el filtrado y el valor del pH se leyó directamente en la escala del potenciómetro.

Humedad: se determinó por el método 925,09 de la AOAC (1997) para la determinación de humedad por pérdida de peso después de secar las muestras a $105^{\circ} \mathrm{C}$ por $4 \mathrm{~h}$.

Pérdida de peso: se realizó de acuerdo con la metodología descrita por Nair, Saxena \& Kaur (2018) con ligeras modificaciones. Se registró el peso inicial de los chiles con papel y se almacenaron a temperatura ambiente. El testigo fueron chiles sin el recubrimiento de papel. Los chiles se pesaron cada $24 \mathrm{~h}$ durante 10 días.

Color: los cambios de color se determinaron de acuerdo con la metodología de Valiathan \& Athmaselvi (2018) en las coordenadas CIELab mediante un colorímetro digital (CHN Spec CS-10). Se mostró como a*(- verde, +rojo), b* (-azul, + amarillo $)$ y L* $(0=$ negro, $100=$ blanco $)$. Se tomaron al menos tres mediciones en diferentes áreas del chile.

Contenido de ácido ascórbico: Se llevó a cabo por el método volumétrico de 2,6-diclorofenol-indofenol descrito por Nair et al. (2018).

\section{Análisis estadístico}

Se utilizó un diseño experimental completamente al azar, todas las determinaciones se realizaron al menos por triplicado. Se realizó un análisis de varianza $(\mathrm{p}<0.05)$ y un análisis de medias aplicando la prueba de diferencia mínima significativa de Fisher (DMS), a las propiedades del papel, así como a las principales características fisicoquímicas del chile serrano con la evaluación del papel como envase.

\section{RESULTADOS Y DISCUSIÓN}

Análisis químico proximal

Los resultados del análisis químico proximal del tallo/hoja del maíz (Cuadro II) son similares a los obtenidos por Treviño, Hernández \& Caballero (2011), quienes reportan un contenido de proteína de 7.6 y $9.3 \%$, extracto etéreo de 1.0 y $1.8 \%$, para tallo y hoja de maíz respectivamente. Sin embargo, estos mismos autores también reportaron valores más altos que los encontrados en este trabajo para cenizas de 8.2 y $14.7 \%$. En este estudio no se determinó el contenido de la celulosa, sin embargo, otros autores han reportado que el contenido de celulosa en las hojas varía de 23.6-27.89\% y en el tallo de 27.9-31.50\% (Amador \& Boschini-Figueroa, 2000; Treviño et al., 2011). Por otra parte, Odetoye, Afolabi, Abu Bakar \& Titiloye (2018), reportaron que el contenido de humedad en la cáscara del piñón varía de 1.02 a $10.75 \%$ y las cenizas de 1.22 a $6.81 \%$ para distintos cultivares de piñón, además, obtuvieron $32.5 \%$ de celulosa de la cáscara del piñón mexicano.

\section{Características físicas y químicas de los papeles}

Las muestras de papel con un contenido de celulosa de CCJ:CTM en proporción 10:90 (P3 y P4) fueron las que presentaron el mayor gramaje de papeles elaborados en el presente estudio, como se puede ver en el Cuadro III; lo que indica una buena interacción entre los tipos de celulosa situación que favorece el incremento de esta propiedad en el papel. Por otra parte, se observó que la reducción en el contenido de CCJ y la incorporación de las etapas de tamizado y prensado también tuvieron un efecto significativo $(p<0.05)$ en la reducción del gramaje de los papeles.

Se ha definido a los productos con un gramaje menor o igual a $120 \mathrm{~g} / \mathrm{m}^{2}$ como papel, mientras que productos con valores entre $130-250 \mathrm{~g} / \mathrm{m}^{2}$ son denominados cartulina y cartón con valores de gramaje mayor a $250 \mathrm{~g} / \mathrm{m}^{2}$ (Ríos Padilla, 2017). Lo importante de conocer el gramaje se debe a que esta propiedad indica que tan imprimible es el papel (Mengual, 2016). Con los resultados obtenidos en este estudio se define al producto P5 como papel, mientras que a los productos P1, P2 y P4 se les considera cartulinas y al P3 como cartón.

Cuadro II. Composición químico proximal de las materias primas utilizadas para la elaboración del papel.

\begin{tabular}{|c|c|c|c|}
\hline Componente & \multicolumn{3}{|c|}{ Composición (\%) } \\
\hline & Tallo de maíz & Hoja de maíz & Cáscara de piñón mexicano \\
\hline Humedad & $10.09 \pm 0.11$ & $8.04 \pm 0.03$ & $11.76 \pm 0.81$ \\
\hline Extracto etéreo & $2.96 \pm 0.64$ & $0.74 \pm 0.02$ & $1.93 \pm 0.21$ \\
\hline Cenizas & $8.42 \pm 0.16$ & $2.53 \pm 0.10$ & $5.38 \pm 0.43$ \\
\hline Fibra cruda & $35.65 \pm 0.66$ & $49.88 \pm 2.15$ & $53.80 \pm 2.26$ \\
\hline Proteína & $6.82 \pm 0.92$ & $8.76 \pm 1.05$ & $4.05 \pm 0.35$ \\
\hline ELN* & $36.06 \pm 0.97$ & $30.05 \pm 3.20$ & $23.08 \pm 3.18$ \\
\hline ELN* Extracto libre de nitrógeno. Los valores son la media de al menos tres repeticiones \pm la desviación estándar.
\end{tabular}


Cuadro III. Características físicas y químicas de los papeles obtenidos con las diferentes proporciones de fibras de Jatropha curcas (CCJ)- Maíz (CTM) y papel comercial (P6).

\begin{tabular}{|c|c|c|c|c|c|c|}
\hline & P1 & P2 & P3 & P4 & P5 & P6 \\
\hline Gramaje $\left(\mathrm{g} / \mathrm{m}^{2}\right)$ & $159.8 \pm 9.3^{\mathrm{c}}$ & $150.3 \pm 7.2^{\mathrm{c}}$ & $287.1 \pm 10.4^{\mathrm{a}}$ & $184.0 \pm 9.5^{\mathrm{b}}$ & $97.0 \pm 1.0^{\mathrm{d}}$ & $46.7 \pm 1.2$ \\
\hline Dureza $(\mathrm{MPa})$ & $1,991 \pm 11^{\mathrm{c}}$ & $720 \pm 4^{\mathrm{e}}$ & $4,237 \pm 2^{\mathrm{a}}$ & $1,144 \pm 2^{\mathrm{d}}$ & $3,112 \pm 9^{\mathrm{b}}$ & $2330 \pm 10$ \\
\hline Espesor $(\mathrm{mm})$ & $0.75 \pm 0.02^{\mathrm{b}}$ & $0.50 \pm 0.01^{\mathrm{d}}$ & $1.00 \pm 0.01^{\mathrm{a}}$ & $0.66 \pm 0.01^{\mathrm{c}}$ & $0.25 \pm 0.01^{\mathrm{e}}$ & $0.06 \pm 0.01$ \\
\hline Humedad $(\%)$ & $1.80 \pm 0.04^{\mathrm{e}}$ & $2.80 \pm 0.12^{\mathrm{c}}$ & $7.86 \pm 0.34^{\mathrm{a}}$ & $3.94 \pm 0.59^{\mathrm{b}}$ & $1.50 \pm 0.12^{\mathrm{d}}$ & $6.65 \pm 0.15$ \\
\hline Cenizas $(\%)$ & $1.48 \pm 0.14^{\mathrm{d}}$ & $2.57 \pm 0.17^{\mathrm{b}}$ & $2.01 \pm 0.24^{\mathrm{bc}}$ & $3.42 \pm 0.44^{\mathrm{a}}$ & $2.35 \pm 0.30^{\mathrm{b}}$ & $5.38 \pm 0.15$ \\
\hline Absorción (s) & $4.21 \pm 0.07^{\mathrm{e}}$ & $7.51 \pm 0.25^{\mathrm{c}}$ & $12.54 \pm 0.08^{\mathrm{a}}$ & $8.83 \pm 0.77^{\mathrm{b}}$ & $5.59 \pm 0.46^{\mathrm{d}}$ & $4.92 \pm 0.16$ \\
\hline
\end{tabular}

Los valores son la media de al menos tres repeticiones \pm la desviación estándar. Letras distintas dentro de cada fila indican diferencia significativa $(\mathrm{p}<0.05)$.

Por otra parte, como se puede observar en el Cuadro III, la dureza de los papeles elaborados disminuyó cuando el contenido de CCJ fue de $20 \%$ (P2), pero una reducción de hasta $10 \%$ favoreció el incremento de la dureza, ya que el papel P3 presentó la mayor fuerza para ser perforado. Además, el tamizado redujo la resistencia de $\mathrm{P} 4$ a romperse, mientras que la adición de la etapa de prensado en P5, incrementó su dureza, indicando nuevamente interacción entre los dos tipos de fibra de CCJ y CTM. La dureza es importante y depende de la unión entre las fibras, para que sea fácilmente rasgado el papel se requiere cierta unión mínima entre las fibras, las cuales se deben fundamentalmente a puentes de hidrógeno (Villareal Jiménez, 2004; Torres, 2019). El tamizado separa las fibras de acuerdo con el número de malla, por lo que no efectuar esta etapa hace que las fibras de distintas longitudes se crucen y el papel sea más denso como sucedió con P3.

Nuevamente la proporción 10:90 CCJ-CTM (P3) fue la que presentó el mayor espesor respecto a los demás papeles. En el caso del papel P4 y P5 disminuyó el espesor del papel debido a las etapas de tamizado y tamizado/prensado respectivamente.

Los resultados obtenidos en este estudio para el espesor $(\mathrm{P} 5=0.25)$ son similares a los reportados por Jeetah \& Jaffur (2021), quienes documentan espesores de 0.267-0.276 mm en hojas de papel elaborado a partir de la cáscara del coco y papel reciclado. Por lo tanto, el espesor depende de la composición fibrosa, de los tratamientos en la refinación y compresión durante la fabricación, así como de la porosidad final del papel (Jesús Díaz, 2020).

En este estudio, el gramaje está relacionado con el espesor, por lo que a mayor gramaje mayor espesor y viceversa, como sucede para P3 y P5, que presentaron el mayor y menor valor en el gramaje y el espesor respectivamente. Se han reportado tendencias similares, donde el gramaje aumentó de 145.39 a $162.08 \mathrm{~g} / \mathrm{m} 2$ con espesores de 0.61 a $0.77 \mathrm{~mm}$ respectivamente, en el papel elaborado con hojas de Pandan (Juliano \& Tayo, 2020).

En el caso del papel comercial (P6), presentó el menor valor de espesor debido al proceso industrial al que es sometido durante su elaboración, ya que la hoja de papel atraviesa por una serie de grandes prensas cuya función es eliminar el agua y reducir el espesor de la hojas (Bajpai, 2015).

Por otra parte, al reducir el contenido de humedad, el gramaje será menor y la compactación de las fibras y la fuerza que ejerce sobre ellas reduce el espesor (Casey, 1991). Además, la celulosa puede absorber agua y humedad del ambiente debido a su naturaleza hidrofílica, provocando el hinchamiento de sus fibras y cambios en el espesor (Ashori \& Sheshmani, 2010; Das, 2017; Kabir, Wang, Lau \& Cardona, 2012; Samyn, 2013). Esto se ve reflejado en el P3, el cual al tener un alto contenido de humedad presentó mayor espesor. Aunado a esto, el contenido de humedad está relacionado con el prensado del papel, ya que se libera el agua durante la compresión como ocurre con el P5, que presentó el nivel más bajo de contenido de agua. Los valores de humedad reportados en este estudio son similares a los obtenidos por Jirukkakul (2018), quien reporta valores de humedad entre $6-13 \%$ en el papel elaborado con los tallos y las hojas de las plantas de banano y cuya presentación es con una película laminada.

Como se observa en la Figura 1 el papel comercial(P6) presentó una estructura más compacta seguida del papel elaborado con maíz al 100\% (P1), donde también hay una buena unión entre las fibras, sin embargo, respecto a la textura, el papel elaborado con maíz al $100 \%$ presentó pelusa en la superficie, un hecho por lo general indeseable, no solo por el desgaste y desprendimiento de la fibra, sino también por ser un riesgo físico en el envasado de alimentos. Por otro lado, el tamaño de la fibra del piñón mexicano afecta el espesor de la hoja (P2), sin embargo, hay mayor uniformidad entre las fibras de $\mathrm{P} 3$ cuando se redujo la proporción a $90-10 \%$, respecto a la proporción $80-20 \%$ del 


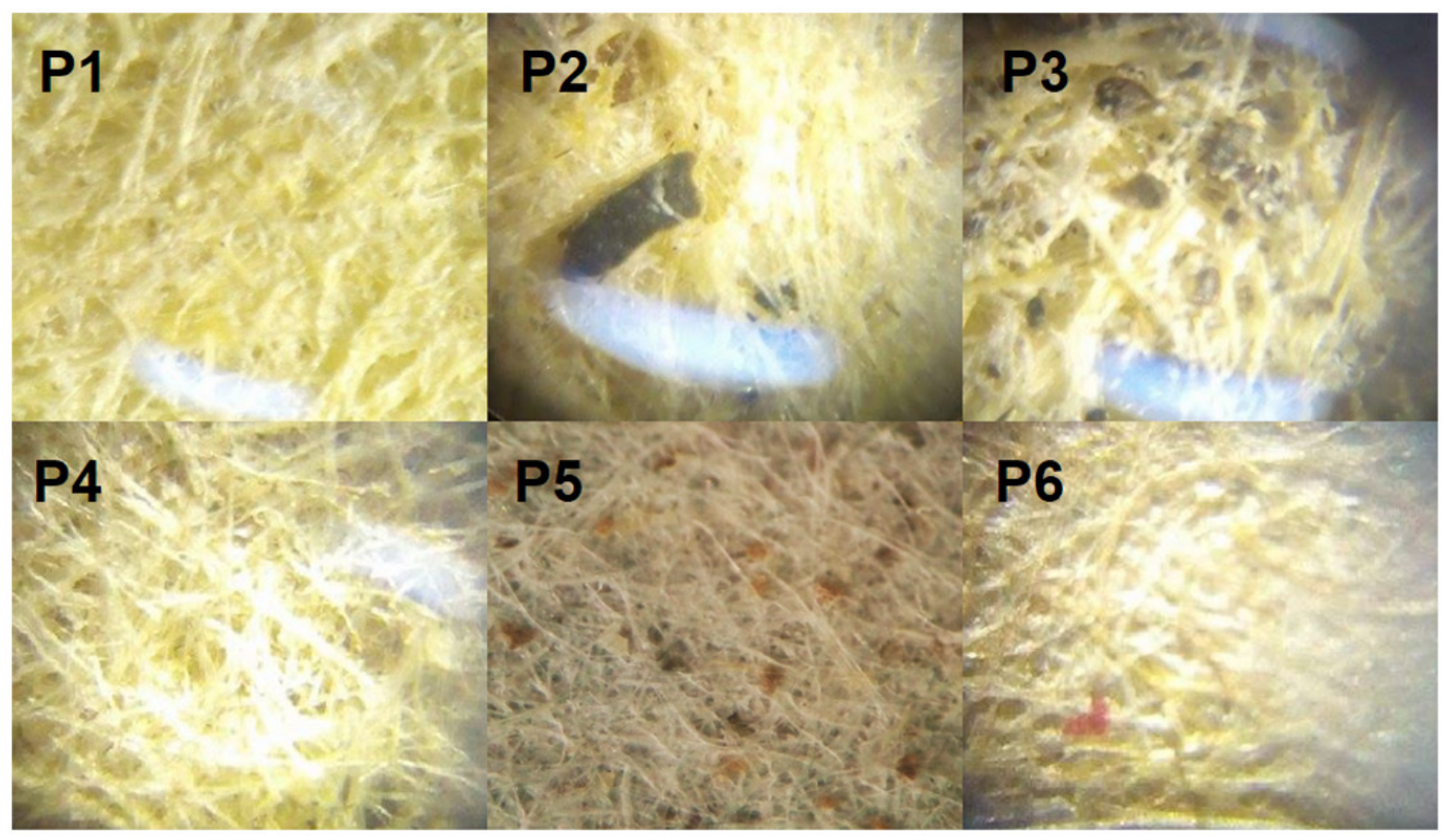

Figura 1. Observación microscópica de las fibras de los papeles elaborados con maíz y Jatropha curcas con un objetivo 4/0.10. Las imágenes corresponden a proporciones de CCJ-CTM de 0-100\% (P1), 20-80\% (P2), 10-90\% (P3), 10-90\% tamizado (P4), 5-95\% tamizado y prensado (P5) y papel comercial (P6).

$\mathrm{P} 2$, además en éste se vio un espacio mayor entre las fibras y con mayor contenido de humedad retenida entre sus espacios.

Cuando se incorporó la etapa de tamizado al papel con la proporción $90-10 \%$ el tamaño de las fibras fue uniforme (P4), sin embargo, se incrementó el espesor. Finalmente, las etapas de tamizado y prensado en el P5 generaron mayor unión entre las fibras, uniformidad y un espesor adecuado para implementarse como envase.

Los papeles elaborados con las diferentes proporciones de CCJ y CTM presentaron menores contenidos de ceniza (1.48-3.42\%) que el papel comercial evaluado (5.38\%). Si el contenido de cenizas excede al 5\%, y su apariencia es densa y compacta, podrá aceptarse como prueba positiva de que el papel ha sido rellenado o recubierto con pigmento. Los papeles elaborados para una resistencia muy elevada (por ejemplo, los papeles para envolver y como cartones para envases) deben tener un bajo contenido de cenizas, porque la presencia de minerales tiende a reducir la resistencia del papel (Casey, 1991).

Adicionar CCJ incrementó significativamente el tiempo de absorción del agua (5.59-12.54 s) en las muestras de papel al compararlas con el papel elaborado con 100\% de CTM (4.21 s). Los resultados obtenidos son mayores a los reportados por Amode \& Jeetah (2021), quienes obtuvieron valores de absorción del agua de 0.7-1.4 s en papel a partir de fibras de cáñamo de "Mauricio". La absorción está relacionada con el espesor, como se observa en P2, P3, P4 y P5 que presentaron mayor tiempo de absorción y mayor espesor. El tiempo de absorción del agua es importante, ya que se debe evitar en mayor o menor medida dependiendo del tipo de papel. Un estudio realizado por Vaithanomsat et al. (2021) demostró que al agregar como recubrimiento el poli(3-hidroxibutirato) aumentaron significativamente los valores de absorción del agua en el papel elaborado con la fibra de la hoja de la piña de hoja (50 s).

Los procesos y maquinarias que se utilizan para dar mayor acabado al papel no fueron implementados en la elaboración del que se presenta en este trabajo, por lo que no resultó apropiado comparar los valores obtenidos con los del papel comercial. La fabricación de papeles con características especiales como es el caso que nos ocupa, puede orientar hacia la aplicación específica de envases para alimentos que se dañan antes de ser consumidos.

De acuerdo con los resultados anteriores, se seleccionó el papel P5, elaborado con 5\% de CCJ y 95\% de CTM con las etapas de tamizado y prensado, ya que presentó características similares al papel comercial (dureza de 2,330 MPa, un espesor de 0.06 mm y absorción de agua de $4.92 \mathrm{~s}$ ). 
Propiedades fisicoquímicas del chile serrano en envase de papel

La firmeza de los chiles disminuyó con el paso de los días (Figura 2A), sin embargo, la firmeza presentó diferencia significativa a partir del día $6(\mathrm{p}<0.05)$ entre los usados como testigo y los protegidos con papel P5 como envase, con valores más altos de firmeza en los protegidos con papel. Este comportamiento es similar a lo reportado por Valiathan \& Athmaselvi (2018), quienes usaron un recubrimiento de goma arábiga sobre chiles serranos con diferentes tiempos de inmersión. Los valores de firmeza oscilan para el control entre $2.26 \mathrm{~N}$ y $0.85 \mathrm{~N}$, y para los recubiertos entre $2.62 \mathrm{~N}$ y $1.46 \mathrm{~N}$ al inicio y al final de la prueba con un tiempo de inmersión de un minuto.

Miranda-Molina et al. (2019) atribuyen que la pérdida de firmeza es un efecto combinado de la degradación de los compuestos poliméricos en las paredes celulares y la pérdida de turgencia ocasionada por la transpiración, manifiesta en mayor cantidad durante su estudio con chiles serranos almacenados a $25^{\circ} \mathrm{C}$ que a $8^{\circ} \mathrm{C}$, ya que a mayor temperatura existe mayor tasa de procesos metabólicos. La firmeza es un indicador de la madurezy depende
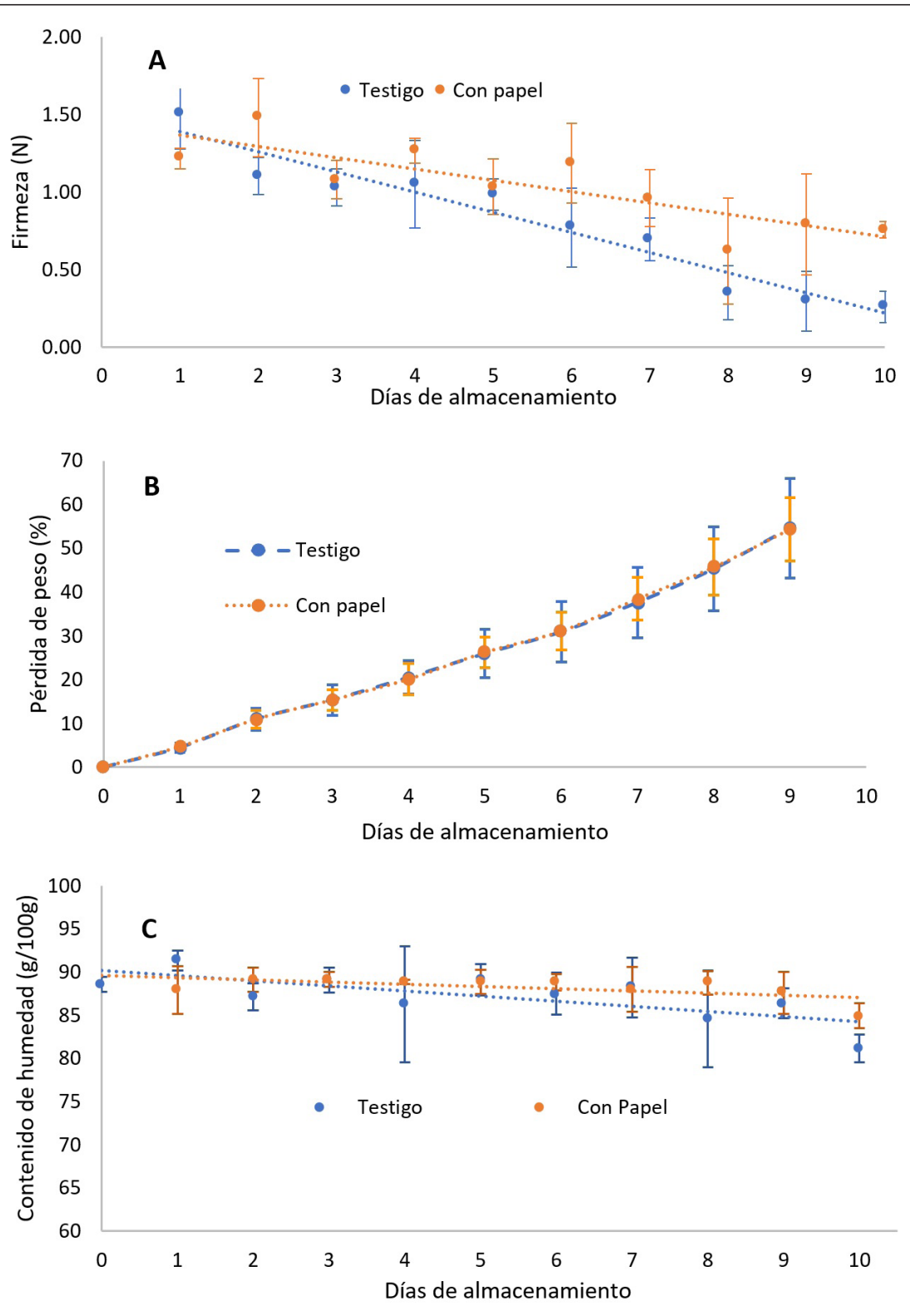

Figura 2. A: firmeza de los chiles serranos testigo y los cubiertos con papel, B: pérdida de peso en los chiles serranos testigo y los cubiertos con papel y $C$ : cambios en el contenido de humedad de los chiles serranos testigo y los cubiertos con papel. Los valores se expresan como el promedio de al menos tres mediciones. Las barras indican desviación estándar. 
del estado de la fruta en el momento de la recolección, de la temperatura y de la forma de almacenamiento, aunque también puede relacionarse con el color externo (Monsalve, Velásquez \& Tórres, 2007). El uso de empaques dentro de las prácticas postcosecha por parte de pequeños agricultores han resultado favorables (Flores-López, Cerqueira, de Rodríguez \& Vicente, 2016). En este estudio, el uso del papel como envase para los chiles serranos les confirió una mayor firmeza, importante como atributo de textura para su aceptación entre los consumidores (Chitravathi et al., 2020).

Por otra parte, el desarrollo del color y la pérdida de firmeza están relacionados con el contenido de oxígeno generado en una atmósfera, donde se demoran los cambios a menores concentraciones de este gas; al ser el papel una barrera física que disminuye la penetración del oxígeno dentro del envase de papel da por resultado menor pérdida de firmeza (Gómez $\&$ Camelo, 2002).

\section{Acidez titulable y pH}

La acidez titulable mostró una tendencia de cambio similar entre ambas muestras de chile (envasados con papel y el testigo) con una variación entre 0.18 a $0.22 \%$ para los chiles testigo y 0.13 a $0.18 \%$ para los chiles envasados. Los valores de acidez encontrados en este trabajo son mayores a los reportados por Miranda-Molina et al. (2019), quienes evaluaron el efecto de tres temperaturas en el contenido de acidez, entre otros parámetros, durante el almacenamiento del chile serrano $(0.04-0.09 \%$ a $25^{\circ} \mathrm{C}$ ). Sin embargo, en las muestras de chile envasadas con papel P5 y el testigo, la acidez titulable presentó diferencias significativas entre ambos tratamientos desde el día 1 hasta el día $10(\mathrm{p}<0.05)$. Se ha reportado que la acidez titulable en los chiles se expresa como ácido cítrico y presenta una tendencia de incremento durante el periodo de almacenamiento. El incremento de la acidez titulable puede asociarse con la actividad enzimática de la pectinmetilesterasa (PME). Por el contrario, la reducción de los valores de la acidez titulable podría deberse al aumento en la tasa de respiración y a la reducción de los ácidos orgánicos (Ortiz, 2013; Tsegay, Tesfaye, Mohammed \& Yirga, 2013).

Respecto al $\mathrm{pH}$, se mantuvo sin grandes variaciones durante los días de almacenamiento en un intervalo de 5.94 para el primer día y al día diez presentó un valor de 5.37 para los chiles testigo, valores similares se encontraron para los almacenados en el envase de papel P5, con un valor de $\mathrm{pH}$ de 5.92 el primer día y de 5.61 al día diez. Los valores obtenidos en este estudio son similares a los documentados por Martínez, Curros, Bermúdez, Carballo \& Franco (2007), quienes reportan valores de pH entre 4.6 y 5.5 en chiles verdes.

Valores bajos de $\mathrm{pH}$ indican presencia deácido cítrico y permiten una vida de anaquel más amplia, ya que actuará a nivel fisiológico en el fruto como barrera fisiológica natural frente a la acción microbiana (Domene \& Segura, 2014; Martínez et al., 2007). De esta manera, los ácidos presentes en el chile influyen en el sabor de los alimentos, en la estabilidad microbiana y en la conservación general de su calidad.

\section{Determinación de la humedad y pérdida de peso}

El contenido de la humedad en los alimentos, es un aspecto clave desde el punto de vista económico y de preservación de la calidad, puesto que con la pérdida de agua o deshidratación existe la disminución del peso fresco y afecta la apariencia, la textura y en algunos casos el sabor, siendo además un índice del grado de maduración de los productos vegetales y en muchos casos el primer criterio de calidad para la decisión de compra y consumo (Jiang et al., 2018; Smith, Stommel, Fung, Wang \& Whitaker, 2006).

Los contenidos de humedad encontrados en este trabajo fueron de $20^{\circ} \mathrm{C}$ y $63.4 \%$ de HR, ligeramente superiores a lo reportado para "chiles siete caldos" (85.30-86.23\%) cultivados bajo condiciones de cielo abierto y en casa sombra (Solís-Marroquín et al., 2017) y valores de $71.23-82.97 \%$ de humedad en otras variedades de Capsicum annuum (Antonio, 2010). La humedad es un parámetro importante en la calidad de los chiles, sin embargo, la mayoría de los estudios reportados evalúan la pérdida de peso.

Para las muestras de chile en un envase de papel P5 y los testigos, se presentó un contenido de humedad similar durante los días de almacenamiento, como se observa en la Figura 2B, donde los chiles serranos cubiertos con papel P5 mantuvieron el porcentaje de humedad estable en un intervalo de $90-85 \%$, a diferencia de los testigo que presentaron mayor variación entre los días de almacenamiento (barras de error de la Figura 2B) con una humedad de $92-81 \%$. Ante esto, los chiles cubiertos con papel lograron mantener un mayor contenido de humedad que los chiles testigo al día 10 .

De acuerdo con la ley de difusión de Fick, la transpiración de un fruto se describe como la diferencia de presión de vapor de agua entre la fruta y el aire circundante y por la resistencia a la evaporación del agua a través de la epidermis del fruto (Díaz-Pérez, Muy-Rangel \& Mascorro, 2007). Otros factores que influyen en la tasa de transpiración son las propiedades intrínsecas del producto fresco como: la morfología, la relación superficie/volumen, la etapa de madurez, las lesiones; y las propiedades extrínsecas entre las que están la temperatura de almacenamiento, la humedad relativa, el movimiento del aire y la presión atmosférica (Sousa-Gallagher, Mahajan \& Mezdad, 2013).

A medida que el vapor de agua pasa de la superficie del producto al aire, se crea una fina atmósfera más saturada alrededor del fruto y disminuye la diferencia de presión del vapor productoaire; sin embargo, un ligero movimiento de aire disipa esta 
atmósfera reemplazándola por aire menos saturado, con menor presión de vapor y mayor capacidad de absorción de agua. Además, cuanto mayor sea la superficie expuesta al aire de un producto, en relación con su peso, mayor será su capacidad de transpiración (Gregori, 2007).

Por lo tanto, es probable que el papel mantuvo una atmósfera saturada alrededor de los chiles y con esto la transpiración fue menor; diferente a los testigo, pues, al no estar protegidos por una barrera de papel, el movimiento de vapor de agua provocó inestabilidad en la transpiración y por consecuencia una variación en la humedad. Esto coincide con la firmeza de los chiles cubiertos con el papel P5, ya que al permanecer la humedad en un rango estable (Figura 2B), la pérdida de firmeza fue menor (Figura 2A). Por el contrario, los chiles testigo tuvieron una pérdida de firmeza mayor debido a la inestabilidad del contenido de humedad.

La pérdida de peso de los chiles (Figura 2C) fue lineal en ambos tratamientos y sin diferencia significativa $(\mathrm{p}<0.05)$ entre los tratamientos. La pérdida de peso de los productos hortícolas se debe principalmente a la transpiración inducida por un déficit de presión de vapor entre el tejido interno y el ambiente, siendo una característica común de estos productos (Miranda-Molina et al., 2019).

El tipo y tamaño de la fruta, las prácticas de huerta, el estado de maduración, las condiciones meteorológicas y ambientales, las técnicas de la cosecha, la composición de la atmósfera del producto dentro y fuera del empaque y la duración del almacenamiento entre otros factores, influyen en la pérdida de agua de las frutas y verduras (Lufu, Ambaw \& Opara, 2020; Quiroz, 2016).

Algunos alimentos como los tomates, las berenjenas y los pimientos soportan mayor pérdida de agua a través de su cáliz, ya que la cutícula cerosa minimiza la pérdida de agua a través de la superficie de la piel. La cosecha de frutas y verduras en su óptima madurez reduce las pérdidas de agua postcosecha durante el periodo de almacenamiento. La temperatura es uno de los factores más importantes en la pérdida de humedad en los productos frescos (Lufu et al., 2020).

Los resultados son similares a los reportados por Rao et al. (2011), quienes atribuyen que la pérdida de peso del producto aumenta progresivamente con el tiempo de almacenamiento provocando marchitamiento y una reducción de su valor en el mercado así como el rechazo del consumidor.

\section{Vitamina C}

Los valores obtenidos en este estudio fueron de $60.71 \mathrm{y}$

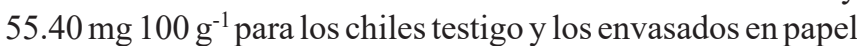
respectivamente, en los puntos máximos del contenido de esta vitamina, son mayores a los reportados para el chile serrano por Xavier \& Pérez-Gálvez (2016) (44.9 mg 100g-1). Otros investigadores han reportado contenidos de ácido ascórbico para chiles de 48.27 a $23.47 \mathrm{mg} 100 \mathrm{~g}^{-1}$ y para los chiles con

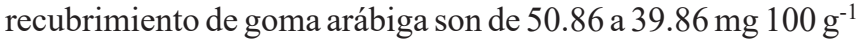
(Valiathan \& Athmaselvi, 2018).

Los contenidos de vitamina $\mathrm{C}$ en los chiles testigo y los almacenados con papel $\mathrm{P} 5$ presentaron diferencia significativa $(p<0.05)$, sin embargo, la tendencia fue similar en ambas muestras hasta el día 6 (Figura 3), con un incremento al paso de los días de almacenamiento, hasta un máximo de $60.71 \mathrm{mg}$

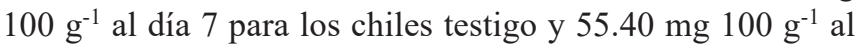
día 8 para los envasados con papel P5. Sin embargo, se puede observar que en los almacenados con papel P5 los valores de la vitamina $\mathrm{C}$ al día 10 fueron de $48.72 \mathrm{mg} 100 \mathrm{~g}-1$, similares a los encontrados entre los días 6 y 9 (49.5-51.2 mg $100 \mathrm{~g}-1)$, mientras que los testigo disminuyeron sus valores de $41.95 \mathrm{mg}$ 100 g-1 al día 10. Este comportamiento ha sido atribuido a que el ácido ascórbico en productos vegetales produce valores máximos inmediatamente antes de la maduración y luego disminuye como resultado de la actividad enzimática (Panigrahi et al., 2017).

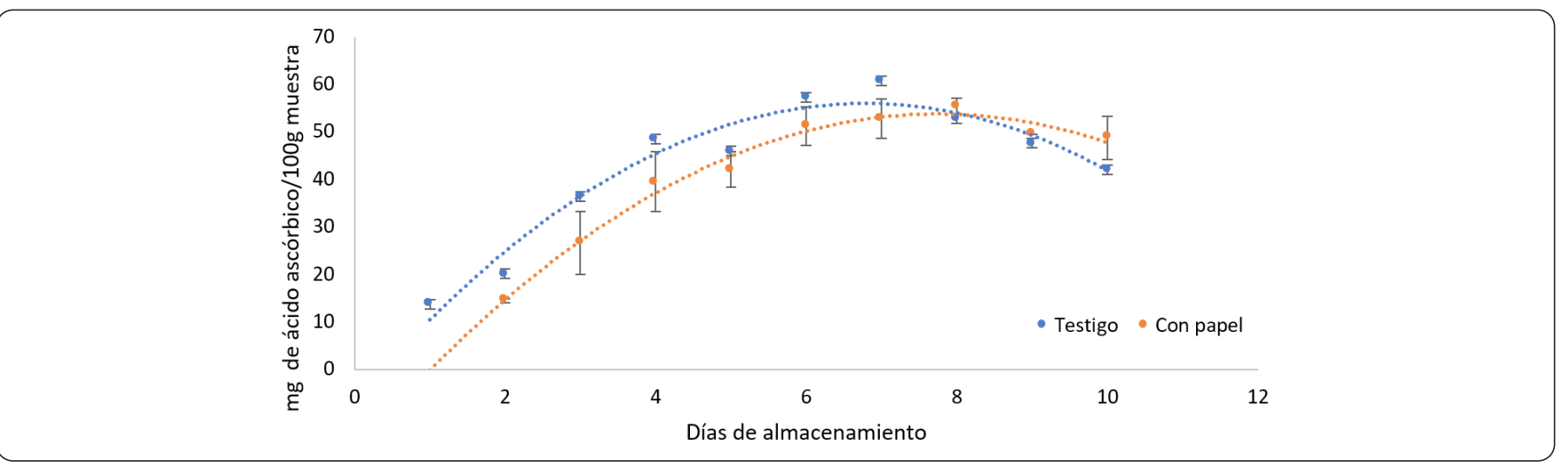

Figura 3. Vitamina $C$ en chiles testigo y chiles envasados con papel P5. Los valores se expresan como el promedio de al menos tres mediciones. Las barras indican desviación estándar. 
El contenido de la vitamina $\mathrm{C}$ se ve afectado por diversos factores como son las variaciones genotípicas, las condiciones climáticas previas a la cosecha, el estado de maduración y los métodos de manipulación postcosecha, por ejemplo la luz, la temperatura, la concentración de oxígeno, el pH, la actividad enzimática y la humedad(Castillo-Téllez, Pilatowsky-Figueroa, López-Vidaña, Sarracino-Martínez \& Hernández-Galvez, 2017; Chitravathi et al., 2020; Herbig \& Renard, 2017; Sapei \& Hwa, 2014; Toledo, Ueda, Imahori \& Ayaki, 2003; Valiathan \& Athmaselvi, 2018).

Por otra parte, el envase podría crear un ambiente hipóxico con una concentración de $\mathrm{CO}_{2}$ modificada debido al proceso de respiración del chile, produciendo una reducción de la vitamina $\mathrm{C}$. Se ha documentado que altas concentraciones de $\mathrm{CO}_{2}$ en la atmósfera de almacenamiento degrada a esta vitamina, probablemente debido a la activación de la ascorbato peroxidasa. Esta tendencia se ha reportado en rodajas de kiwi recién cortado, en manzanas, en grosellas rojas, en fresas y en pimiento almacenados en atmósferas con concentraciones elevadas de $\mathrm{CO}_{2}$, también se ha documentado en la col rizada envasada en una atmósfera modificada. De esta manera, la degradación de la vitamina $\mathrm{C}$ puede variar entre los productos según los niveles de $\mathrm{CO}_{2}$, la temperatura y la duración del almacenamiento (Boerzhijin, Makino, Hirai, Sotome \& Yoshimura, 2020; Lee \& Kader, 2000; Shin et al., 2008).

También se ha reportado que la degradación de la vitamina C está relacionada con el contenido de humedad, por lo que a mayor contenido de humedad mayor degradación (Hiatt, Ferruzzi, Taylor, \& Mauer, 2011). Por lo tanto, los chiles envasados mostraron valores de humedad ligeramente mayores respecto a los testigo (Figura $2 \mathrm{~B}$ ), por lo que pudo haber existido mayor degradación de la vitamina en estas condiciones de humedad.

\section{Color}

En las coordenadas de color L, a* y b* para los chiles testigo y los envasados en papel P5 obtenidas en el presente estudio, existió diferencia significativa $(\mathrm{p}<0.05)$ a partir del día 2 y hasta el día 10 y entre los días de almacenamiento con el mismo tratamiento. Como se observa en los cuadros IV y V, se mantuvo una retención del color verde $\left(-\mathrm{a}^{*}\right)$ en los chiles envasados con papel P5 durante los 10 días de almacenamiento, atribuible a la tasa de respiración reducida por la atmósfera creada dentro del envase de papel, como ha sido discutido anteriormente. Ha sido explicado que el cambio de valor de a* (de negativo a positivo) se debe a la síntesis de carotenoides (transformación de cloroplasto a cromoplastos) que ocurre durante el proceso de senescencia (Chitravathi et al., 2015; Valiathan \& Athmaselvi, 2018). En los chiles testigo se observó un cambio de color verde $\left(-\mathrm{a}^{*}\right)$ a color rojo $\left(+\mathrm{a}^{*}\right)$ a partir del día 7 , también se observó un cambio en las coordenadas de color amarillo $(+b)$, e incrementado en función de los días de almacenamiento comparado con los chiles envasados en papel P5.

El color constituye una de las cualidades sensoriales más apreciables a simple vista $y$, en consecuencia, desempeña un papel muy importante en las características de calidad de las hortalizas (Gil, 2010). En particular, los chiles serranos se consumen frescos y con coloración verde, por lo que la vida útil después de la cosecha se ha definido como el tiempo para cambiar de una tonalidad verde a roja/naranja o el tiempo requerido para perder su consistencia turgente debido a la transpiración (Miranda-Molina et al., 2019). La firmeza y el color son parámetros fáciles de medir para estimar la madurez de un fruto, ya que con el proceso de maduración se genera un ablandamiento y cambios de color del fruto hasta alcanzar la máxima calidad comestible. Con este proceso de maduración se aumenta la desorganización de los tejidos del fruto, la degradación de los pigmentos y el pudrimiento del

\section{Cuadro IV. Determinación del color por CIE-La*b*.}

\begin{tabular}{|c|c|c|c|c|c|c|c|c|c|c|}
\hline \multicolumn{11}{|c|}{ Con Papel } \\
\hline Día & 1 & 2 & 3 & 4 & 5 & 6 & 7 & 8 & 9 & 10 \\
\hline $\mathrm{L}$ & $11.33 \pm 0.58^{\mathrm{a}, \mathrm{A}}$ & $10.67 \pm 0.58^{\mathrm{a}, \mathrm{B}}$ & $11.67 \pm 0.58^{\mathrm{a}, \mathrm{A}}$ & $11.67 \pm 0.58^{\mathrm{a}, \mathrm{A}}$ & $10.33 \pm 0.58^{\mathrm{a}, \mathrm{B}}$ & $8.33 \pm 1.15^{\mathrm{a}, \mathrm{C}}$ & $9.33 \pm 0.58^{\mathrm{a}, \mathrm{C}}$ & $9.67 \pm 0.58^{b, C}$ & $9.33 \pm 0.58^{\mathrm{b}, \mathrm{C}}$ & $9.33 \pm 0.58^{\mathrm{b}, \mathrm{C}}$ \\
\hline$b^{*}$ & $7.00 \pm 1.73^{\mathrm{a}, \mathrm{A}}$ & $5.67 \pm 0.58^{\mathrm{a}, \mathrm{BC}}$ & $7.67 \pm 0.58^{\mathrm{a}, \mathrm{A}}$ & $6.33 \pm 1.15^{\mathrm{a}, \mathrm{AB}}$ & $6.67 \pm 0.57^{\mathrm{a}, \mathrm{AB}}$ & $4.33 \pm 1.15^{\mathrm{a}, \mathrm{BC}}$ & $6.33 \pm 0.35^{\mathrm{b}, \mathrm{B}}$ & $5.33 \pm 0.58^{\mathrm{b}, \mathrm{C}}$ & $5.00 \pm 1.00^{\mathrm{b}, \mathrm{C}}$ & $5.33 \pm 0.58^{\mathrm{b}, \mathrm{C}}$ \\
\hline$a^{*}$ & $-9.33 \pm 1.53^{\mathrm{a}, \mathrm{C}}$ & $-6.67 \pm 0.58^{\mathrm{a}, \mathrm{B}}$ & $-6.00 \pm 1.73^{\mathrm{a}, \mathrm{B}}$ & $-6.00 \pm 1.00^{\mathrm{a}, \mathrm{B}}$ & $-6.33 \pm 1.53^{\mathrm{a}, \mathrm{B}}$ & $-5.00 \pm 2.00^{\mathrm{a}, \mathrm{B}}$ & $-6.00 \pm 1.00^{\mathrm{a}, \mathrm{B}}$ & $23.33 \pm 9.29^{\mathrm{a}, \mathrm{A}}$ & $34.00 \pm 6.24^{\mathrm{a}, \mathrm{A}}$ & $32.33 \pm 4.93^{\mathrm{a}, \mathrm{A}}$ \\
\hline$b^{*}$ & $7.00 \pm 1.73^{\mathrm{a}, \mathrm{B}}$ & $2.67 \pm 0.58^{\mathrm{b}, \mathrm{D}}$ & $3.33 \pm 1.15^{\mathrm{b}, \mathrm{CD}}$ & $3.00 \pm 0.00^{\mathrm{b}, \mathrm{D}}$ & $4.50 \pm 0.50^{\mathrm{b}, \mathrm{CD}}$ & $3.00 \pm 1.00^{\mathrm{a}, \mathrm{D}}$ & $4.67 \pm 0.58^{\mathrm{a}, \mathrm{C}}$ & $28.00 \pm 1.00^{\mathrm{a}, \mathrm{A}}$ & $28.00 \pm 8.19^{\mathrm{a}, \mathrm{A}}$ & $35.33 \pm 7.37^{\mathrm{a}, \mathrm{A}}$ \\
\hline
\end{tabular}


Cuadro V. Cambio de coloración del chile serrano almacenado con y sin papel a temperatura ambiente.

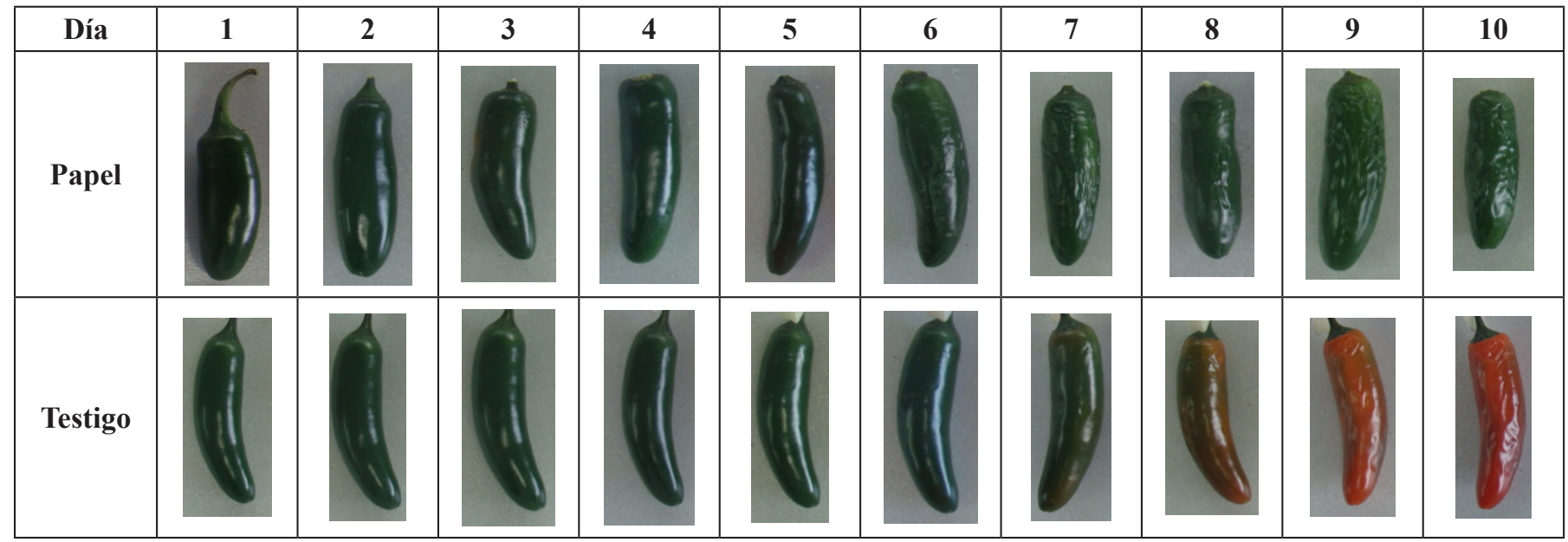

producto (Castellanos, Polanía \& Herrera, 2016; Panigrahi et al., 2017).

Finalmente, la aceptación o rechazo a un alimento está relacionado con una serie de atributos visuales, olfativos y táctiles (Wang et al., 2019). El deterioro de estos atributos en los productos frescos se ve afectado durante la cadena de suministro y las condiciones de almacenamiento. Sin embargo, estas pérdidas postcosecha se pueden llegar a reducir con barreras físicas que retrasen la senescencia y mantengan la calidad del producto (Aguilar-Méndez et al., 2012; Macheka, Spelt, van der Vorst \& Luning, 2017).

\section{Conclusiones}

El envase de papel elaborado a partir de residuos fibrosos de hojas/tallos de maíz y de la cáscara del piñón mexicano presentó características deseables para ser implementado en la conservación de los chiles serranos debido a que los valores de gramaje, dureza y espesor son similares a otros materiales comerciales.

El papel protegió al chile favoreciendo los valores de firmeza, humedad, color y vitamina $C$, conservando la calidad comestible del producto, sin modificar la tendencia de cambio en los valores de $\mathrm{pH}$, acidez titulable y pérdida de peso.

\section{Agradecimientos}

Alejandra Linares Castañeda agradece al programa BEIFI de la SIP del Instituto Politécnico Nacional por el apoyo otorgado. Agradecemos también el apoyo de la SECTEI de la Ciudad de México por el financiamiento mediante el proyecto CMSECITI/085/2017.

\section{CONFLICTO DE INTERÉS}

Los autores declaran que no hay conflictos de interés.

\section{REFERENCIAS}

Abrantes, S., Amaral, M. E., Costa, A. P. \& Duarte, A. P. (2007). Cynara cardunculus L. alkaline pulps:Alternatives fibres for paper and paperboard production. Bioresource Technology, 98(15), 2873-2878. https://doi.org/10.1016/J. BIORTECH.2006.09.052

Adel, A. M., El-Gendy, A. A., Diab, M. A., Abou-Zeid, R. E. \& El-Zawawy, W. K. (2016). Microfibrillated cellulose from agricultural residues. Part I: Papermaking application. Industrial Crops and Products, 93, 161-174. https://doi. org/10.1016/J.INDCROP.2016.04.043

Adetunji, C. O., Ojediran, J. O., Adetunji, J. B. \& Owa, S. O. (2019). Influence of chitosan edible coating on postharvest qualities of Capsicum annum L. during storage in evaporative cooling system . Croatian Journal of Food Science and Technology, 11(1), 59-66. https://doi. org/10.17508/cjfst.2019.11.1.09

Aguilar-Méndez, M. A., San Martín-Martínez, E., EspinozaHerrera, N. L., Sánchez-Flores, M., Cruz-Orea, A. \& Ramírez-Ortíz, M. E. (2012). Caracterización y aplicación de películas a base de gelatina-carboximetilcelulosa para la preservación de frutos de guayaba. Superficies y Vacio, 25(1), 1-7. Retrieved from http://www.scielo. org. $\mathrm{mx} /$ scielo.php? script $=$ sci_arttext\&pid $=\mathrm{S} 1665$ 35212012000100001\&lng=es\&tlng $=$ es

Amador, A. L. \& Boschini-Figueroa, C. (2000). Fenología productiva y nutricional de maíz para la producción de forraje. Agronomía Mesoamericana, 11(1), 171-177.

Amode, N. S. \& Jeetah, P. (2021). Paper Production from Mauritian Hemp Fibres. Waste and Biomass Valorization, 12(4), 1781-1802. https://doi.org/10.1007/s12649-02001125-y

Anguiano Barrales, J. (2010). Comparación en la respuesta fisiológica en plantas de chile bajo el efecto de tres temperaturas nocturnas. Tesis de maestría. Universidad 
Autónoma de Nuevo León, 136. Retrieved from http:// eprints.uanl.mx/2043/1/1080190958.pdf

Antonio, E. (2010). Composición química próximal y evaluación de la capacidad antioxidante del chile de agua (Capsicum annuum L.). Tesis de maestría. Universidad Autónoma Metropolitana, 76. Retrieved from. Retrieved from http://148.206.53.233/tesiuami/UAMI15479.pdf

AOAC. (1997). Association of Official Analytical Chemists. Official Methods of Analysis, Vol. 16, 3rd revision, Gaithersburg, MD.

Ashori, A. \& Sheshmani, S. (2010). Hybrid composites made from recycled materials: Moisture absorption and thickness swelling behavior. Bioresource Technology, 101(12), 4717-4720. https://doi.org/10.1016/j.biortech.2010.01.060

Bajpai, P. (2015). Basic Overview of Pulp and Paper Manufacturing Process. In Green Chemistry and Sustainability in Pulp and Paper Industry, 11-39. https:// doi.org/10.1007/978-3-319-18744-0_2

Boerzhijin, S., Makino, Y., Hirai, M. Y., Sotome, I. \& Yoshimura, M. (2020). Effect of perforation-mediated modified atmosphere packaging on the quality and bioactive compounds of soft kale (Brassica oleracea L. convar. acephala (DC) Alef. var. sabellica L.) during storage. Food Packaging and Shelf Life, 23, 100427. https://doi. org/10.1016/j.fpsl.2019.100427

Bras, J., Hassan, M. L., Bruzesse, C., Hassan, E. A., El-Wakil, N. A. \& Dufresne, A. (2010). Mechanical, barrier, and biodegradability properties of bagasse cellulose whiskers reinforced natural rubber nanocomposites. Industrial Crops and Products, 32(3), 627-633. https://doi.org/10.1016/J. INDCROP.2010.07.018

Casey, J. (1991). Pulpa y papel. Química y tecnología química. Vol. 1. Limusa, México

Castellanos, D. A., Polanía, W. \& Herrera, A. O. (2016). Development of an equilibrium modified atmosphere packaging (EMAP) for feijoa fruits and modeling firmness and color evolution. Postharvest Biology and Technology, 120, 193-203. https://doi.org/10.1016/j. postharvbio.2016.06.012

Castillo-Téllez, M., Pilatowsky-Figueroa, I., López-Vidaña, E. C., Sarracino-Martínez, O. \& Hernández-Gálvez, G. (2017). Dehydration of the red chilli (Capsicum annuum L., costeño) using an indirect-type forced convection solar dryer. Applied Thermal Engineering, 114, 1137-1144. https://doi.org/10.1016/j.applthermaleng.2016.08.114

Chitravathi, K., Chauhan, O. P. \& Raju, P. S. (2014). Postharvest shelf-life extension of green chillies (Capsicum annuum L.) using shellac-based edible surface coatings. Postharvest Biology and Technology, 92, 146-148. https://doi. org/10.1016/j.postharvbio.2014.01.021

Chitravathi, K., Chauhan, O. P. \& Raju, P. S. (2015). Influence of modified atmosphere packaging on shelf-life of green chillies (Capsicum annuum L.). Food Packaging and Shelf Life, 4, 1-9. https://doi.org/10.1016/j.fpsl.2015.02.001
Chitravathi, Kallepalli, Chauhan, O. P. \& Kizhakkedath, J. (2020). Shelf life extension of green chillies (Capsicum annuum L.) using passive modified atmosphere packaging and gamma irradiation. Journal of Food Processing and Preservation, 44(8), e14622. https://doi.org/10.1111/ jfpp. 14622

Das, S. (2017). Mechanical and water swelling properties of waste paper reinforced unsaturated polyester composites. Construction and Building Materials, 138,469-478. https:// doi.org/10.1016/j.conbuildmat.2017.02.041

Díaz-Pérez, J. C., Muy-Rangel, M. D. \& Mascorro,A. G. (2007). Fruit size and stage of ripeness affect postharvest water loss in bell pepper fruit (Capsicum annum L.). Journal of the Science of Food and Agriculture, 87(1), 68-73. https://doi. org/10.1002/jsfa.2672

Domene, M. A. \& Segura, M. (2014). Parámetros de calidad interna de hortalizas y frutas en la industria agroalimentaria. Negocios Agroalimentario Cooper Cajamar Fichas Transf, $\mathbf{5}, 1-18$.

Eckhoff, S. R., Paulsen, M. R. \& Yang, S. C. (2003). Maize. In B. Caballero, P. Finglas \& F. Toldra (Ed.). Encyclopedia of Food Sciences and Nutrition (Second edition). Academic Press, 3647-3653, New York. https://doi.org/10.1016/B012-227055-X/00725-2

Egüés, I., Sanchez, C., Mondragon, I. \& Labidi, J. (2012). Effect of alkaline and autohydrolysis processes on the purity of obtained hemicelluloses from corn stalks. Bioresource Technology, 103(1), 239-248. https://doi.org/10.1016/J. BIORTECH.2011.09.139

Emana, B., Afari-Sefa, V., Nenguwo, N., Ayana, A., Kebede, D. \& Mohammed, H. (2017). Characterization of pre- and postharvest losses of tomato supply chain in Ethiopia. Agriculture \& Food Security, 6(1), 3. https://doi. org/10.1186/s40066-016-0085-1

Fan, C. \& Zhang, Y. (2018). Adsorption isotherms, kinetics and thermodynamics of nitrate and phosphate in binary systems on a novel adsorbent derived from corn stalks. Journal of Geochemical Exploration, 188, 95-100. https:// doi.org/10.1016/J.GEXPLO.2018.01.020

Florentino-Ramos, E., Villa-Ruano, N., Hidalgo-Martínez, D., Ramírez-Meraz, M., Méndez-Aguilar, R., Velásquez-Valle, R., Zepeda-Vallejo, L. G., Pérez-Hernández, N. \& BecerraMartínez, E. (2019). 1H NMR-based fingerprinting of eleven Mexican Capsicum annuum cultivars. Food Research International, 121, 12-19. https://doi.org/10.1016/j. foodres.2019.03.025

Flores-López, M. L., Cerqueira, M. A., de Rodríguez, D. J. \& Vicente, A. A. (2016). Perspectives on utilization of edible coatings and nano-laminate coatings for extension of postharvest storage of fruits and vegetables. Food Engineering Reviews, 8(3), 292-305. https://doi. org/10.1007/s12393-015-9135-x

Gil, Á. (2010). Tratado de nutrición, Tomo II: Composición y calidad nutritiva de los alimentos. Madrid. Editorial 
Panamericana.

Gómez, P. \& Camelo, A. (2002). Calidad postcosecha de tomates almacenados en atmósferas controladas. Horticultura Brasileira, 20, 38-43. Retrieved from http:// www.scielo.br/scielo.php?script=sci_arttext\&pid=S010205362002000100007\&nrm=iso

Gregori, G. (2007). La transpiración de frutas y verduras. 17o Symposium International PHYTOMA.

Han, Q., Gao, X., Zhang, H., Chen, K., Peng, L. \& Jia, Q. (2019). Preparation and comparative assessment of regenerated cellulose films from corn (Zea mays) stalk pulp fines in DMAc/LiCl solution. Carbohydrate Polymers, 218, 315-323. https://doi.org/10.1016/j.carbpol.2019.04.083

Hedayati, S. \& Niakousari, M. (2015). Effect of coatings of silver canoparticles and gum arabic on physicochemical and microbial properties of green bell pepper ( Capsicum annuum ). Journal of Food Processing and Preservation, 39(6), 2001-2007. https://doi.org/10.1111/jfpp.12440

Herbig, A. L. \& Renard, C. M. G. C. (2017). Factors that impact the stability of vitamin $\mathrm{C}$ at intermediate temperatures in a food matrix. Food Chemistry, 220, 444-451. https://doi. org/10.1016/j.foodchem.2016.10.012

Hernández-López, G., Ventura-Aguilar, R. I., Correa-Pacheco, Z. N., Bautista-Baños, S. \& Barrera-Necha, L. L. (2020). Nanostructured chitosan edible coating loaded with $\alpha$-pinene for the preservation of the postharvest quality of Capsicum annuum L. and Alternaria alternata control. International Journal of Biological Macromolecules, 165, 1881-1888. https://doi.org/10.1016/j.ijbiomac.2020.10.094

Hiatt, A. N., Ferruzzi, M. G., Taylor, L. S. \& Mauer, L. J. (2011). Deliquescence behavior and chemical stability of vitamin $\mathrm{C}$ forms (ascorbic acid, sodium ascorbate, and calcium ascorbate) and blends. International Journal of Food Properties, 14(6), 1330-1348. https://doi. org/10.1080/10942911003650338

Iewkittayakorn, J., Khunthongkaew, P., Wongnoipla, Y., Kaewtatip, K., Suybangdum, P. \& Sopajarn, A. (2020). Biodegradable plates made of pineapple leaf pulp with biocoatings to improve water resistance. Journal of Materials Research and Technology, 9(3), 5056-5066. https://doi.org/10.1016/j.jmrt.2020.03.023

Jarabo, R., Monte, M. C., Fuente, E., Santos, S. F. \& Negro, C. (2013). Corn stalk from agricultural residue used as reinforcement fiber in fiber-cement production. Industrial Crops and Products, 43, 832-839. https://doi.org/10.1016/J. INDCROP.2012.08.034

Jeetah, P. \& Jaffur, N. (2021). Coconut husk, a lignocellulosic biomass, as a promising engineering material for non-wood paper production. Journal of Natural Fibers, 1-15. https:// doi.org/10.1080/15440478.2021.1889428

Jesús Díaz, L. G. (2020). Obtención y evaluación de la pulpa para papel del endocarpio del fruto de Mauritia flexuosa (Aguaje). Universidad Nacional de Ucayali. Retrieved from http://repositorio.unu.edu.pe/bitstream/handle/
UNU/4325/UNU FORESTAL 2020 T LIANA-JESUS. 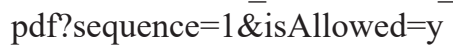

Jiang, J., Cen, H., Zhang, C., Lyu, X., Weng, H., Xu, H. \& He, Y. (2018). Nondestructive quality assessment of chili peppers using near-infrared hyperspectral imaging combined with multivariate analysis. Postharvest Biology and Technology, 146, 147-154. https://doi.org/10.1016/j. postharvbio.2018.09.003

Jirukkakul, N. (2018). Physical properties of banana stem and leaf papers laminated with banana film. Walailak Journal of Science and Technology (WJST), 16(10), 753-763. https:// doi.org/10.48048/wjst.2019.3471

Juliano, M.A. L. \& Tayo, L. L. (2020). Utilization of pandan leaf fibers (Pandanus simplex merr.) for the production of paper. IOP Conference Series: Earth and Environmental Science, 563(1). https://doi.org/10.1088/1755-1315/563/1/012012

Kabir, M. M., Wang, H., Lau, K. T. \& Cardona, F. (2012). Chemical treatments on plant-based natural fibre reinforced polymer composites: An overview. Composites Part B: Engineering, 43(7), 2883-2892. https://doi.org/10.1016/j. compositesb.2012.04.053

Kumar, A. \& Sharma, S. (2008). An evaluation of multipurpose oil seed crop for industrial uses (Jatropha curcas L.): A review. Industrial Crops and Products, 28(1), 1-10. https:// doi.org/10.1016/J.INDCROP.2008.01.001

Lavoine, N., Desloges, I., Dufresne, A. \& Bras, J. (2012). Microfibrillated cellulose - Its barrier properties and applications in cellulosic materials: Areview. Carbohydrate Polymers, 90(2), 735-764. https://doi.org/10.1016/J. CARBPOL.2012.05.026

Lee, S. K. \& Kader, A. A. (2000). Preharvest and postharvest factors influencing vitamin $\mathrm{C}$ content of horticultural crops. Postharvest Biology and Technology, 20(3), 207-220. https://doi.org/10.1016/S0925-5214(00)00133-2

Lim, B. Y., Shamsudin, R., Baharudin, B. T. H. T. \& Yunus, R. (2015). A review of processing and machinery for Jatropha curcas L. fruits and seeds in biodiesel production: Harvesting, shelling, pretreatment and storage. Renewable and Sustainable Energy Reviews, 52, 991-1002. https:// doi.org/10.1016/J.RSER.2015.07.077

Liu, C., Wang, X., Lin, F., Zhang, H. \& Xiao, R. (2018). Structural elucidation of industrial bioethanol residual lignin from corn stalk: A potential source of vinyl phenolics. Fuel Processing Technology, 169, 50-57. https://doi. org/10.1016/j.fuproc.2017.09.008

Liu, Y., Xie, J., Wu, N., Ma, Y., Menon, C. \& Tong, J. (2019). Characterization of natural cellulose fiber from corn stalk waste subjected to different surface treatments. Cellulose, 26(8), 4707-4719. https://doi.org/10.1007/s10570-01902429-6

Lufu, R., Ambaw, A. \& Opara, U. L. (2020). Water loss of fresh fruit: Influencing pre-harvest, harvest and postharvest factors. Scientia Horticulturae, 272(2020), 109519. https:// doi.org/10.1016/j.scienta.2020.109519 
Luo, Z., Li, P., Cai, D., Chen, Q., Qin, P., Tan, T. \& Cao, H. (2017). Comparison of performances of corn fiber plastic composites made from different parts of corn stalk. Industrial Crops and Products, 95, 521-527. https://doi. org/10.1016/j.indcrop.2016.11.005

Macheka, L., Spelt, E., van der Vorst, J. G. A. J. \& Luning, P. A. (2017). Exploration of logistics and quality control activities in view of context characteristics and postharvest losses in fresh produce chains: A case study for tomatoes. Food Control, 77, 221-234. https://doi.org/10.1016/J. FOODCONT.2017.02.037

Martínez, S., Curros, A., Bermúdez, J., Carballo, J. \& Franco, I. (2007). The composition of Arnoia peppers (Capsicum annuum L.) at different stages of maturity. International Journal of Food Sciences and Nutrition, 58(2), 150-161. https://doi.org/10.1080/09637480601154095

Mazumdar, P., Singh, P., Babu, S., Siva, R. \& Harikrishna, J. A. (2018). An update on biological advancement of Jatropha curcas L.: New insight and challenges. Renewable and Sustainable Energy Reviews, 91, 903-917. https://doi. org/10.1016/J.RSER.2018.04.082

Mengual, I. (2016). Papeles, efectos y manipulados. Retrieved from http://umh2127.edu.umh.es/wp-content/uploads/ sites/906/2016/10/7.1.-Papeles-efectos-manipulados-.pdf

Miranda-Molina, F. D., Valle-Guadarrama, S., Guerra-Ramírez, D., Arévalo-Galarza, M. D. L., Pérez-Grajales, M. \& ArtésHernández, F. (2019). Quality attributes and antioxidant properties of Serrano chili peppers (Capsicum annuum L.) affected by thermal conditions postharvest. International Food Research Journal, 26(2), 1889-1898.

Monsalve, D. M. O., Velásquez, H. J. C. \& Tórres, I. D. A. (2007). Determinación de la fuerza de la fractura superficial $\mathrm{y}$ fuerza de firmeza en frutas de lulo (Solanum quitoense $\mathrm{x}$ Solanum hirtum). Revista Facultad Nacional de Agronomía Medellin, 60(2), 4163-4178.

Nair, M. S., Saxena, A. \& Kaur, C. (2018). Characterization and antifungal activity of pomegranate peel extract and its use in polysaccharide-based edible coatings to extend the shelf-life of capsicum (Capsicum annuum L.). Food and Bioprocess Technology, 11(7), 1317-1327.https://doi. org/10.1007/s11947-018-2101-x

NMX-N-001-SCFI. (2011). Industrias de celulosa y papel Determinación del gramaje o peso base del papel, cartoncillo y cartón (peso por unidad de área) - Método de prueba (Cancela a la NMX-N-001-SCFI-2005). Retrieved from http://www.economia-nmx.gob.mx/normas/nmx/2010/ nmx-n-001-scfi11.pdf

NMX-N-016-SCFI. (2011). Industrias de celulosa y papelDeterminación de humedad de papeles y cartones por secado en estufa - Método de prueba. Retrieved from http://www.economia-nmx.gob.mx/normas/nmx/2010/ nmx-n-016-scfil1.pdf

NMX-N-098-SCFI. (2014). Industrias de celulosa y papel - Determinación de la absorción de agua por el papel medium mediante la absorción de la gota-Método de prueba. Retrieved from http://www.economia-nmx.gob. mx/normas/nmx/2010/nmx-n-098-scfi-2014.pdf

Odetoye, T. E., Afolabi, T. J., Abu Bakar, M. S. \& Titiloye, J. O. (2018). Thermochemical characterization of Nigerian Jatropha curcas fruit and seed residues for biofuel production. Energy, Ecology and Environment, 3(6), 330-337. https://doi.org/10.1007/s40974-018-0104-0

Ortiz, H. G. (2013). Efectos del acolchado plástico y la fertilización química y biológica sobre la calidad y vida de anaquel de pimiento, asistida con recubrimiento biodegradable de poliacetato de vinilo-alcohol polivinílico. Tesis de Maestría. México. Centro de Investigación en Química Aplicada, 154. Retrieved from https://ciqa. repositorioinstitucional.mx/jspui/handle/1025/37

Pandey, V. C., Singh, K., Singh, J. S., Kumar, A., Singh, B. \& Singh, R. P. (2012). Jatropha curcas: A potential biofuel plant for sustainable environmental development. Renewable and Sustainable Energy Reviews, 16, 2870-2883. https://doi. org/10.1016/j.rser.2012.02.004

Panigrahi, J., Gheewala, B., Patel, M., Patel, N. \& Gantait, S. (2017). Gibberellic acid coating: A novel approach to expand the shelf-life in green chilli (Capsicum annuum L.). Scientia Horticulturae, 225(April), 581-588. https:// doi.org/10.1016/j.scienta.2017.07.059

Pechanova, O. \& Pechan, T. (2017). Proteomics as a tool to understand maize biology and to improve maize crop. Chapter 3. In M. L. Colgrove (Ed.). Proteomics in Food Science: from farm to fork, Academic Press, 35-56, Berlin. https://doi.org/10.1016/B978-0-12-804007-2.00003-5

Porat, R., Lichter,A., Terry, L.A., Harker, R.\& Buzby, J. (2018). Postharvest losses of fruit and vegetables during retail and in consumers' homes: Quantifications, causes, and means of prevention. Postharvest Biology and Technology, 139, 135149. https://doi.org/10.1016/j.postharvbio.2017.11.019

Quiroz, J.A.(2016). Dinámica de la pérdida de peso en hortalizas de hoja durante el almacenamiento. Universidad de Costa Rica. Retrieved from http:/www.ingbiosistemas.ucr.ac.cr/ wp-content/uploads/2016/09/Tesis_Dinamica_perdida peso_hojas.pdf

Rao, T. V. R., Gol, N. B. \& Shah, K. K. (2011). Effect of postharvest treatments and storage temperatures on the quality and shelf life of sweet pepper (Capsicum annum L.). Scientia Horticulturae, 132(1), 18-26. https://doi. org/10.1016/j.scienta.2011.09.032

Rios Padilla, A. D. (2017). Producción de papel artesanal a partir de residuos de cáscaras de naranja de las juguerias del Mercado Tahuantinsuyo-Independencia, 2017. Universidad César Vallejo.

Samyn, P. (2013). Wetting and hydrophobic modification of cellulose surfaces for paper applications. Journal of Materials Science, 48(19), 6455-6498. https://doi. org/10.1007/s10853-013-7519-y

Sapei, L. \& Hwa, L. (2014). Study on the kinetics of vitamin C 
degradation in fresh strawberry juices. Procedia Chemistry, 9, 62-68. https://doi.org/10.1016/j.proche.2014.05.008

Sethi, J., Oksman, K., Illikainen, M. \& Sirviö, J. A. (2018). Sonication-assisted surface modification method to expedite the water removal from cellulose nanofibers for use in nanopapers and paper making. Carbohydrate Polymers, 197, 92-99. https://doi.org/10.1016/J.CARBPOL.2018.05.072

Shin, Y., Ryu, J. A., Liu, R. H., Nock, J. F., Polar-Cabrera, K. \& Watkins, C. B. (2008). Fruit quality, antioxidant contents and activity, and antiproliferative activity of strawberry fruit stored in elevated $\mathrm{CO}_{2}$ atmospheres. Journal of Food Science, 73(6), S339-S344.https://doi.org/10.1111/j.17503841.2008.00857.x

Singh, R., Giri, S. K. \& Kotwaliwale, N. (2014). Shelf-life enhancement of green bell pepper (Capsicum annuum L.) under active modified atmosphere storage. Food Packaging and Shelf Life, 1(2), 101-112. https://doi.org/10.1016/j. fps1.2014.03.001

Smith, D. L., Stommel, J. R., Fung, R. W. M., Wang, C. Y. \& Whitaker, B. D. (2006). Influence of cultivar and harvest method on postharvest storage quality of pepper (Capsicum annuum L.) fruit. Postharvest Biology and Technology, 42(3), 243-247. https://doi.org/10.1016/j. postharvbio.2006.06.013

Solís-Marroquín, D., Lecona-Guzmán, C. A., Ruiz-Lau, N., Ocampo, P., Rodas-Trejo, J., Gonzales-Santiago, C., González-Mejía, O. \& Gordillo-Páez, L. (2017). Análisis bromatológico de frutos de chile "siete caldos" (Capsicum annuum) cultivados en condiciones de cielo abierto y casa sombra. Agro productividad, 10(9), 34-40. Retrieved from http://search.ebscohost.com/login.aspx?direct=true $\& \mathrm{db}=\mathrm{f}$ ap\&AN=126130458\&site $=$ ehost-live

Sousa-Gallagher, M. J., Mahajan, P. V. \& Mezdad, T. (2013). Engineering packaging design accounting for transpiration rate: Model development and validation with strawberries. Journal of Food Engineering, 119(2), 370-376. https://doi. org/10.1016/j.jfoodeng.2013.05.041

Toledo, M. E. A., Ueda, Y., Imahori, Y. \& Ayaki, M. (2003). L-ascorbic acid metabolism in spinach (Spinacia oleracea L.) during postharvest storage in light and dark. Postharvest Biology and Technology, 28(1), 47-57. https://doi. org/10.1016/S0925-5214(02)00121-7

Torres, M.A. (2019). Obtención de celulosa a partir de la cáscara de cacao ecuatoriano (Theobroma cacao L.) mediante hidrólisis térmica para la elaboración de pulpa de papel. Universidad Central Del Ecuador.

Treviño, J., Hernández, T. \& Caballero, R. (2011). Estudio del valor nutritivo de las hojas y tallo del maíz híbrido de tallo azucarado E-10. Instituto de Alimentación y Productividad Animal (C.S.I.C).
Tsegay, D., Tesfaye, B., Mohammed, A. \& Yirga, H. (2013). Effects of harvesting stage and storage duration on postharvest quality and shelf life of sweet bell pepper (Capsicum annuum L.) varieties under passive refrigeration system. International Journal of Biotechnology and Molecular Biology Research, 4(7), 98-104.

Vaithanomsat, P., Kongsin, K., Trakunjae, C., Boonyarit, J., Jarerat, A., Sudesh, K. \& Chollakup, R. (2021). Biosynthesized poly(3-Hydroxybutyrate) on Coated pineapple leaf fiber papers for biodegradable packaging application. Polymers, 13(11), 1733. https://doi. org/10.3390/polym 13111733

Valiathan, S. \& Athmaselvi, K. A. (2018). Gum arabic based composite edible coating on green chillies. International Agrophysics, 32(2), 193-202. https://doi.org/10.1515/ intag-2017-0003

Villa-Ruano, N., Ramírez-Meraz, M., Méndez-Aguilar, R., Zepeda-Vallejo, L. G., Álvarez-Bravo,A., Pérez-Hernández, N. \& Becerra-Martínez, E. (2019). 1H NMR-based metabolomics profiling of ten new races from Capsicum annuum cv. serrano produced in Mexico. Food Research International, 119, 785-792. https://doi.org/10.1016/j. foodres.2018.10.061

Villarreal Jiménez, L. A. (2004). Uso de lodo de papel y arena sílica para la fabricación de ladrillos y tabla roca. Universidad de las Américas Puebla.

Wang, Q. J., Mielby, L. A., Junge, J. Y., Bertelsen, A. S., Kidmose, U., Spence, C. \& Byrne, D. V. (2019). The role of intrinsic and extrinsic sensory factors in sweetness perception of food and beverages: A review. Foods, 8, 211. https://doi. org/10.3390/foods8060211

Xavier,A.A. O.\& Pérez-Gálvez,A. (2016). Peppers and Chilies. Encyclopedia of Food and Health, 301-306. https://doi. org/10.1016/B978-0-12-384947-2.00533-X

Xia, F., Liu, H., Lu, J., Lv, Y., Zhai, S., An, Q., Cheng, Y. \& Wang, H. (2019). An integrated biorefinery process to produce butanol and pulp from corn straw. Industrial Crops and Products, 140(2019), 111648. https://doi.org/10.1016/j. indcrop. 2019.111648

Xing, Y., Li, X., Xu, Q., Yun, J., Lu, Y.\& Tang, Y. (2011). Effects of chitosan coating enriched with cinnamon oil on qualitative properties of sweet pepper (Capsicum annuum L.). Food Chemistry, 124(4), 1443-1450. https://doi.org/10.1016/j. foodchem.2010.07.105

Yousefi, H., Faezipour, M., Hedjazi, S., Mousavi, M. M., Azusa, Y. \& Heidari, A. H. (2013). Comparative study of paper and nanopaper properties prepared from bacterial cellulose nanofibers and fibers/ground cellulose nanofibers of canola straw. Industrial Crops and Products, 43(1), 732-737. https://doi.org/10.1016/j.indcrop.2012.08.030 